Japan. J. Math.

Vol. 23, No. 2, 1997

\title{
Class-group problems for cubic number fields
}

\author{
By Stéphane Louboutin \\ (Received July 3, 1995) \\ (Revised April 2, 1996) \\ (from Nagoya Mathematical Journal)
}

\section{Notations}

When $\mathbf{K}$ is a number field, we let $D_{\mathbf{K}}, d_{\mathbf{K}}, \operatorname{Reg}_{\mathbf{K}}, h_{\mathbf{K}}$ and $e_{\mathbf{K}}$ be its discriminant, the absolute value of its discriminant, its regulator, its class number and the exponent of its ideal class group, respectively. An integral ideal of $\mathbf{K}$ is an ideal of its ring of algebraic integers. An integral ideal $\mathbf{I}$ of $\mathbf{K}$ is said to be non trivial if there does not exist any rational integer $n$ such that $\mathbf{I}=(n)$. Throughout this paper we let $\operatorname{Min}_{\mathbf{K}}$ denote the least norm of the non trivial principal integral ideals of $\mathbf{K}$.

The generalized Riemann hypothesis $G R H_{\mathbf{K}}$ asserts that the Dedekind zeta function of the number field $\mathbf{K}$ is zero-free in the half-plane $\Re(s)>\frac{1}{2}$. Let $\mathbf{N}$ be a normal number field. Under the assumption of $G R H_{\mathbf{N}}$, there exists a prime $p \leq 70 \log ^{2} d_{\mathbf{N}}$ which splits completely in $\mathbf{N}$ (see [Oes, Th. 4]). Moreover, let $\mathbf{L}$ be a quadratic number field. Under the assumption of $G R H_{\mathbf{L}}$, there exist a prime $p \leq 3 \log ^{2} d_{\mathbf{L}}$ which is inert in $\mathbf{L} / \mathbb{Q}$ and a prime $p \leq 2 \log ^{2} d_{\mathbf{L}}$ which does not split in $\mathbf{L} / \mathbb{Q}$ ( see [Bac, Th. 2 and Th. 3]).

\section{Introduction}

We prove that there are exactly 4 pure cubic fields $\mathbf{K}_{m}=\mathbb{Q}\left(\sqrt[3]{d_{m}}\right)$ (with $d_{m}=m^{3}+1 \geq 2$ cube-free) which have ideal class groups of exponents $\leq 3$ (see Theorem 9). Now, assume that the generalized Riemann hypothesis holds. We prove that (i) the exponents of the ideal class groups of the pure cubic number fields $\mathbf{K}_{m}=\mathbb{Q}\left(\sqrt[3]{d_{m}}\right)$ (with $d_{m}=m^{3}+1 \geq 2$ square-free) tend to infinity with the absolute values of their discriminants (see Theorem 3), (ii) the exponents of the ideal class groups of Ishida's real cubic number fields tend to infinity with the absolute values of their discriminants (see Theorem 12), and (iii) we determine all the Ishida's cubic number fields with ideal class groups of exponents $\leq 3$ (see 
Theorem 13). We point out that we do not know how to prove that (i) assuming the generalized Riemann hypothesis then the exponents of the ideal class groups of the pure cubic number fields $\mathbf{K}_{m}=\mathbb{Q}\left(\sqrt[3]{d_{m}}\right)$ (with $d_{m}=m^{3}+1 \geq 2$ cube-free) tend to infinity with the absolute values of their discriminants, and (ii) without assuming any generalized Riemann hypothesis, then there are only finitely many Ishida's cubic number fields with ideal class groups of exponents 2 or 3 .

We conclude this section by giving the following result which will prove that we will be working with two infinite families $\mathbf{K}_{m}$ and $\mathbf{K}_{l}$ of non-normal cubic number fields:

Proposition. (a) (See [Hoo]). If $N(x)$ is the number of positive integers $l \leq x$ with the property that $4 l^{3}+27$ is square-free, we have as $x \rightarrow \infty$

$$
N(x) / x \rightarrow \frac{2}{3}\left(\prod_{p \equiv 5(\bmod 6)}\left(1-\frac{1}{p^{2}}\right)\right)\left(\prod_{\substack{p \equiv 1(\bmod 6) \\ 2^{(p-1) / 3} \equiv 1(\bmod p)}}\left(1-\frac{3}{p^{2}}\right)\right)=0.625 \cdots
$$

(b) (See [Ric]). If $N(x)$ is the number of positive integers $m \leq x$ with the property that $d_{m}=m^{3}+1=(m+1)\left(m^{2}-m+1\right)$ is square-free, we have as $x \rightarrow \infty$

$$
N(x) / x \rightarrow \frac{1}{2}\left(\prod_{p \equiv-1(\bmod 6)}\left(1-\frac{1}{p^{2}}\right)\right)\left(\prod_{p \equiv 1(\bmod 6)}\left(1-\frac{3}{p^{2}}\right)\right)=0.425 \cdots
$$

Proof. According to the references these limits do exist and are equal to

$$
\prod_{p}\left(1-\frac{h\left(p^{2}\right)}{p^{2}}\right)
$$

where $h\left(p^{2}\right)$ denotes the number of solution modulo $p^{2}$ of the equations $f(x) \equiv 0$ $\left(\bmod p^{2}\right)$ with $f(X)=4 X^{3}+27$ or $f(X)=X^{3}+1$, respectively.

\section{Lower bounds on exponents of ideal class groups of non-normal cubic number fields}

Lemma 1. Let $\mathbf{K}$ be a number field of prime degree $q$. Let $p$ be a prime positive integer and let $\mathcal{P}$ be a prime ideal of $\mathbf{K}$ lying above $p$. If there exist $e \geq 1$ and $n \geq 1$ two positive integers such that $\mathcal{P}^{e}=(n)$ is trivial, then either $(p)=\mathcal{P}$ is inert in $\mathbf{K}$, or $q$ divides e and $(p)=\mathcal{P}^{q}$ is totally ramified in $\mathbf{K}$.

Proof. Taking norms we get that $n$ is some $p$-power. Using the decomposition of the ideal $(p)$ into a product of prime ideals, we readily get the desired result. 
TheOREM 2. Let $\mathbf{K}$ be a non normal cubic number field. If there exists an unramified prime ideal of $\mathbf{K}$ with norm $p$ a prime, then $e_{\mathbf{K}} \geq \log \mathrm{Min}_{\mathbf{K}} / \log p$. Hence, if the generalized Riemann hypothesis $G R H_{\mathbf{N}}$ holds for the normal closure $\mathbf{N}$ of $\mathbf{K}$, then $e_{\mathbf{K}} \geq \log \operatorname{Min}_{\mathbf{K}} / \log \left(70 \log ^{2} d_{\mathbf{N}}\right) \gg \log \mathrm{Min}_{\mathbf{K}} / \log \log d_{\mathbf{K}}$.

Proof. If $70 \log ^{2}\left|d_{\mathbf{N}}\right|<\left(\operatorname{Min}_{\mathbf{K}}\right)^{1 / e_{\mathbf{K}}}$ then under the assumption of the generalized Riemann hypothesis for $\mathbf{N}$ there exists a prime $p \leq 70 \log ^{2} d_{\mathbf{N}}<\left(\operatorname{Min}_{\mathbf{K}}\right)^{1 / e_{\mathbf{K}}}$ which splits completely in $\mathbf{N}$. Let $\mathcal{P}$ be a prime ideal of $\mathbf{K}$ lying above $p$. Since $\mathcal{P}^{e_{\mathbf{K}}}$ is a non trivial principal integral ideal with norm less than $\operatorname{Min}_{\mathbf{K}}$, we get a contradiction. Hence, we have $70 \log ^{2} d_{\mathbf{N}} \geq\left(\operatorname{Min}_{\mathbf{K}}\right)^{1 / e_{\mathbf{K}}}$ and the desired result (since we have $\left.d_{\mathbf{N}} \leq d_{\mathbf{K}}^{3}\right)$.

\section{Lower bounds for the exponents of the ideal class group of certain pure cubic number fields}

We let $m \geq 1$ be a positive integer. We set $d_{m}=m^{3}+1, \omega_{m}=\sqrt[3]{d_{m}}$ and $\mathbf{K}_{m}=\mathbb{Q}\left(\omega_{m}\right)$. Throughout this section we will always assume that $d_{m}$ is cube-free. In that case, if $d_{m}=a_{m} b_{m}^{2}$ with $a_{m} \geq 1$ and $b_{m}$ square-free and coprime, then

$$
d_{\mathbf{K}_{m}}= \begin{cases}3\left(a_{m} b_{m}\right)^{2} & \text { if } 3 \text { divides } m \\ 27\left(a_{m} b_{m}\right)^{2} & \text { if } 3 \text { does not divide } m\end{cases}
$$

Let $\mathbf{K}$ be real pure cubic number field. In [Lou3] we used the lower bound

$$
h_{\mathbf{K}} \operatorname{Reg}_{\mathbf{K}} \geq \frac{1}{9} \sqrt{d_{\mathbf{K}} / \log d_{\mathbf{K}}}, \quad\left(d_{\mathbf{K}} \geq 3 \cdot 10^{4}\right)
$$

to solve the class number one, two and three problems for $\mathbf{K}_{m}$. Indeed, $\epsilon_{m}=$ $\omega_{m}^{2}+m \omega_{m}+m^{2}$ is a unit of the ring of algebraic integers of $\mathbf{K}_{m}$ and we have $\epsilon_{m} \leq 3 \omega_{m}^{2}=3 d_{m}^{2 / 3} \leq\left(3 d_{\mathbf{K}_{m}}\right)^{2 / 3}$ and $\operatorname{Reg}_{\mathbf{K}_{m}} \leq \log \epsilon_{m}$. Hence, we get

$$
h_{\mathbf{K}_{m}} \geq \frac{1}{6} \sqrt{d_{\mathbf{K}_{m}} / \log ^{3}\left(3 d_{\mathbf{K}_{m}}\right)}, \quad d_{\mathbf{K}_{m}} \geq 3 \cdot 10^{4} .
$$

Therefore, $h_{\mathbf{K}_{m}}$ approaches infinity when $d_{\mathbf{K}_{m}}$ approaches infinity. Here, we let $e_{m}$ denote the exponent of the ideal class group of the pure cubic number field $\mathbf{K}_{m}$. We will give lower bounds on the exponents of the ideal class groups of $\mathbf{K}_{m}$, and then solve the exponent one, two and three class group problems for $\mathbf{K}_{m}$.

2A. Lower bounds on the exponents of the ideal class groups of $\mathbf{K}_{m}$ when $d_{m}$ is square-free.

THEOREM 3. There are infinitely many positive integers $m \geq 1$ such that $d_{m}$ is square-free. For such $m$, under the assumption of the generalized Riemann 
hypothesis for the normal closure $\mathbf{N}_{m}=\mathbb{Q}\left(\sqrt{-3}, \sqrt[3]{d_{m}}\right)$ of $\mathbf{K}_{m}$ we have a conditional explicit lower bound $e_{m} \gg \log d_{\mathbf{K}_{m}} / \log \log d_{\mathbf{K}_{m}}$. Moreover, there are infinitely many positive even integers $m \geq 1$ such that $d_{m}$ is square-free. For such $m$ we have an unconditional explicit lower bound $e_{m} \gg \log d_{\mathbf{K}_{m}}$.

The proof of this Theorem readily follows from Theorem 2, the following Lemma 4 and the fact that if $m$ is even, then there exists an unramified prime ideal of $\mathbf{K}_{m}$ of norm 2 (see the proof of Theorem 7 below).

Lemma 4. Let $d=a b^{2} \geq 2$ be a cube-free positive integer, with $a \geq 1$ and $b \geq 1$ square-free and coprime. Set $\omega=\sqrt[3]{a b^{2}}$ and $\bar{\omega}=\sqrt[3]{a^{2} b}$. Let $\epsilon>1$ be $a$ unit of the order $\mathbf{A}=\mathbf{Z}+\omega \mathbf{Z}+\bar{\omega} \mathbf{Z}$ of the cubic number field $\mathbf{K}=\mathbb{Q}(\sqrt[3]{d})$. If $\alpha \in \mathbf{A}$ then $\alpha$ is associated to some $\beta=x+y \omega+z \bar{\omega} \in \mathbf{A}$ which satisfies $|y| \leq(N \epsilon)^{1 / 3} / \omega$ and $|z| \leq(N \epsilon)^{1 / 3} / \bar{\omega}$, where $N=\left|N_{\mathbf{K} / \mathbf{Q}}(\alpha)\right|$. Hence, if $N<\operatorname{Min}\left(\omega^{3} / \epsilon, \bar{\omega}^{3} / \epsilon\right)$ then $\alpha$ is associated to some rational integer. In particular, if $d_{m}=m^{3}+1$ is square-free then $\operatorname{Min}_{\mathbf{K}_{m}} \geq m / 81$.

Proof. Let $\alpha=x+y \omega+z \bar{\omega}$ be in $\mathbf{A}$ and let $\lambda>0$ be suitably chosen during the proof. Since $\alpha$ and $\alpha \epsilon^{n}$ have the same norm for any rational integer $n$, we may assume that $\alpha$ satisfies $\lambda \leq|\alpha|<\lambda \epsilon$. Now, taking the conjugates of $\alpha$ we get

$$
\left\{\begin{array}{l}
x+y \omega+z \bar{\omega}=\alpha \\
x+y j \omega+z j^{2} \bar{\omega}=\alpha^{\prime} \\
x+y j^{2} \omega+z j \bar{\omega}=\alpha^{\prime \prime} .
\end{array}\right.
$$

Hence, $3 y \omega=\alpha+j^{2} \alpha^{\prime}+j \alpha^{\prime \prime}$ and $3 z \bar{\omega}=\alpha+j \alpha^{\prime}+j^{2} \alpha^{\prime \prime}$. Since $N=|\alpha|\left|\alpha^{\prime}\right|^{2}=$ $|\alpha|\left|\alpha^{\prime \prime}\right|^{2}$, we get $\left|\alpha^{\prime}\right|=\left|\alpha^{\prime \prime}\right| \leq \sqrt{N / \lambda}$, which yields $3|y| \omega \leq f(\lambda)$ and $3|z| \bar{\omega} \leq f(\lambda)$ where $f(\lambda)=\lambda \epsilon+2 \sqrt{N / \lambda}$. The choice $\lambda=(\sqrt{N} / \epsilon)^{2 / 3}$ yields $f(\lambda)=3(N \epsilon)^{1 / 3}$ and the desired result.

Now, asume that $d_{m}=m^{3}+1$ is square-free, let $\mathbf{A}_{\mathbf{K}_{m}}$ be the ring of algebraic integers of $\mathbf{K}_{m}$, and set $\mathbf{A}_{m}=\mathbf{Z}\left[\omega_{m}\right]$. Then $\mathbf{A}_{m}$ has index 3 or 1 in $\mathbf{A}_{\mathbf{K}_{m}}$ (according as $d_{m} \equiv 1(\bmod 9)$ or not) and $\epsilon_{m}=1 /\left(\omega_{m}-m\right)=\omega_{m}^{2}+m \omega_{m}+m^{2}>1$ is a unit of $\mathbf{A}_{m}$. According to the first part of this Lemma 4 , if $\mathbf{I}=(\alpha)$ is a non trivial principal ideal of $\mathbf{A}_{\mathbf{K}_{m}}$, then $\beta=3 \alpha \in \mathbf{A}_{m}$ satisfies

$$
27 N(\mathbf{I})=\left|N_{\mathbf{K}_{m} / \mathbf{Q}}(\beta)\right| \geq \operatorname{Min}\left(\omega_{m}^{3} / \epsilon_{m}, \omega_{m}^{6} / \epsilon_{m}\right)=\omega_{m}^{3} / \epsilon_{m} \geq \omega_{m} / 3 \geq m / 3
$$

Proposition 5. Assume $m \geq 3$ and $d_{m}=m^{3}+1$ square-free. Set $\mathbf{A}_{m}=$ $\mathbf{Z}\left[\omega_{m}\right]$. If $\alpha \in \mathbf{A}_{m}$ then either there exists a rational integer $n$ and a unit $\eta$ of $\mathbf{A}_{m}$ such that $\alpha=n \eta$, or $N=\left|N_{\mathbf{K}_{m} / \mathbf{Q}}(\alpha)\right| \geq 3 m^{2}-3 m+2$. Note that $\mid N_{\mathbf{K}_{m} / \mathbf{Q}}(m-$ $\left.1-\omega_{m}\right) \mid=3 m^{2}-3 m+2$. In particular, if $d_{m} \not \equiv 1(\bmod 9)$ is square-free then $\operatorname{Min}_{\mathbf{K}_{m}}=3 m^{2}-3 m+2$. 
Proof. According to Lemma 4, $\alpha$ is associated to some $\beta=x-y \omega_{m}+z \omega_{m}^{2}$ which satisfies $|y| \leq\left(N \epsilon_{m}\right)^{1 / 3} / \omega_{m}$ and $|z| \leq\left(N \epsilon_{m}\right)^{1 / 3} / \omega_{m}^{2}$. Hence, $N \leq \omega_{m}^{6} / \epsilon_{m}$ implies $|z|<1$, which yields $z=0, \beta=x-y \omega_{m}$ and also implies $|y| \leq \omega_{m}$. Now, $m \geq 3$ implies $|y| \leq \omega_{m} \leq m^{2}$ and $\omega_{m}^{6} / \epsilon_{m}=\left(m^{3}+1\right)^{2}\left(\omega_{m}-m\right) \geq m^{4} / 3 \geq$ $3 m^{2}-3 m+2$. Since we clearly may assume $y \geq 1$, according to the following Lemma we get Proposition 5 .

LEMMA 6 . $\quad m \geq 1, x \neq m y$ and $1 \leq y \leq m^{2}$ imply $\left|N_{\mathbf{K}_{m} / \mathbf{Q}}\left(x-y \omega_{m}\right)\right|=$ $\left|x^{3}-y^{3}\left(m^{3}+1\right)\right| \geq 3 m^{2}-3 m+2$. Note that if $x=m y$ then $\beta=y\left(m-\omega_{m}\right)=-y / \epsilon_{m}$ is associated to the rational integer $y$.

Proof. Set $x=m y+t$. Then $\left|x^{3}-y^{3}\left(m^{3}+1\right)\right|=\left|t^{3}+3 y m t^{2}+3 y^{2} m^{2} t-y^{3}\right|$. First, assume $t \leq-1$ and set $t=-T$. Then $g(T)=T^{3}-3 y m T^{2}+3 y^{2} m^{2} T+y^{3}$ satisfies $g^{\prime}(T)=3(T-y m)^{2} \geq 0$. Hence, $h(T) \geq h(1)=3 y^{2} m^{2}-3 y m+y^{3}+1=g(y)$ with $g^{\prime \prime}(y)=6 m^{2}+6 y \geq 0$, hence with $g^{\prime}(y) \geq g^{\prime}(1)=6 m^{2}-3 m+3 \geq 0$, hence with $g(y) \geq g(1)=3 m^{2}-3 m+2$. Second, asume $t \geq 1$ and set $h(t)=$ $t^{3}+3 y m t^{2}+3 y^{2} m^{2} t-y^{3}$. Then, $h^{\prime}(t)=3 t^{2}+6 y m t+3 y^{2} m^{2}=3(t+y m)^{2} \geq 0$. Hence, $h(t) \geq h(1)=3 y^{2} m^{2}+3 y m+1-y^{3}=g(y)$ with $g^{\prime \prime}(y)=-6 y+6 m^{2} \geq 0$, hence with $g^{\prime}(y) \geq g^{\prime}(1)=6 m^{3}+3 m-3 \geq 0$, which yields $g(y) \geq g(1)=3 m^{2}+3 m>$ $3 m^{2}-3 m+2$.

Theorem 7. Assume that $d_{m}=m^{3}+1 \not \equiv 1(\bmod 9)($ with $m \geq 3)$ is squarefree, and let $e_{m}$ denote the exponent of the ideal class group of $\mathbf{K}_{m}$. Then, $p$ prime, $p$ does not divide $3 d_{m}$ and $p^{e_{m}} \leq 3 m^{2}-3 m+1$ imply $p \equiv 1(\bmod 3)$ and $d_{m}^{(p-1) / 3} \not \equiv 1(\bmod p)$.

Proof. If $p$ does not divides $d_{\mathbf{K}_{m}}$, then [Coh, Corollary 6.4.15] yields

$$
(p)= \begin{cases}\mathcal{P}_{1} \mathcal{P}_{2} & \text { if } p \equiv 2(\bmod 3) \\ \mathcal{P}_{1} \mathcal{P}_{2} \mathcal{P}_{3} & \text { if } p \equiv 1(\bmod 3) \text { and } d_{m}^{(p-1) / 3} \equiv 1(\bmod p) \\ \mathcal{P} & \text { if } p \equiv 1(\bmod 3) \text { and } d_{m}^{(p-1) / 3} \not \equiv 1(\bmod p) .\end{cases}
$$

According to Lemma 1 and Proposition 5 we get the desired result.

REMARKs. There are 28387 values of $m \geq 3$ such that (i) $m \leq 5 \cdot 10^{4}$, (ii) $d_{m} \neq \equiv 1(\bmod 9)$, and (iii) $d_{m}=m^{3}+1$ is square-free, but only 1771 out of them are such that the necessary conditions of Theorem 7 are fulfilled with $e_{m}=3$.

More generally, if $d_{m}=m^{3}+1 \not \equiv 1(\bmod 9)$ is cube-free and if $\mathbf{K}_{m}$ has an ideal class group of exponent $e_{m}$, then $p^{e_{m}}<\operatorname{Min}\left(\omega_{m}^{3} / \epsilon_{m}, \bar{\omega}_{m}^{3} / \epsilon_{m}\right)$ and $p$ does not divide $d_{\mathbf{K}_{m}}$ imply $p \equiv 1(\bmod 3)$ and $d_{m}^{(p-1) / 3} \not \equiv 1(\bmod p)$ (here $d_{m}=a_{m} b_{m}^{2}$ with $a_{m}$ and $b_{m}$ positive, coprime and square-free, $\omega_{m}=\sqrt[3]{a_{m} b_{m}^{2}}$ and $\left.\bar{\omega}_{m}=\sqrt[3]{a_{m}^{2} b_{m}}\right)$.

If we only assume that $d_{m}$ is cube-free, then we cannot give a lower bound on $\operatorname{Min}\left(\omega_{m}^{3} / \epsilon_{m}, \bar{\omega}_{m}^{3} / \epsilon_{m}\right)$ which would enable us to deduce from Theorem 2 that the exponents $e_{m}$ of $\mathbf{K}_{m}$ go to infinity with $d_{\mathbf{K}_{m}}$. 
2B. Solution of the exponent one, two and three class group problems for $\mathbf{K}_{m}$ with $d_{m}$ cube-free.

Lemma 8 (See [Ger, Th. 3.6]). Let $\mathbf{K}=\mathbb{Q}(\sqrt[3]{d})(d \geq 2$ cube-free $)$ be a pure cubic number field, let $\mathbf{N}=\mathbf{K}(\sqrt{-3})$ be its normal closure and let $T$ be the number of distinct prime ideals of $\mathbf{L}=\mathbb{Q}(\sqrt{-3})$ which are ramified in $\mathbf{N} / \mathbf{L}$. Then, the 3 -rank $\rho_{3}$ of the ideal class group of $\mathbf{K}$ is less than or equal to $T-1$. Moreover, we have

$$
T= \begin{cases}r_{d}+2 s_{d}+1 & \text { if } d \not \equiv 0(\bmod 3) \quad \text { and } d \not \equiv \pm 1(\bmod 9) \\ r_{d}+2 s_{d} & \text { otherwise, }\end{cases}
$$

where $r_{d}$ denotes the number of distinct prime divisors of $d$ which are not equal to 1 modulo 3 and $s_{d}$ denotes the number of distinct prime divisors of $d$ which are equal to 1 modulo 3 .

Proof. Write $D_{\mathbf{K}}=-3 f^{2}$ with $f \geq 1$. Then a prime ideal $\mathcal{P}$ of $\mathbf{L}$ is ramified in $\mathbf{N} / \mathbf{L}$ if and only if $\mathcal{P} \cap \mathbf{Z}=p \mathbf{Z}$ where $p$ divides $f$. Let $t_{f}$ denote the number of prime divisors of $f, r_{f}$ the number of prime divisors of $f$ which are not equal to 1 modulo 3 and $s_{f}$ the number of prime divisors of $f$ which are equal to 1 modulo 3. Then $T=r_{f}+2 s_{f}$. Moreover, if $d=a b^{2}$ with $a$ and $b$ coprime and square-free, then $f=a b$ or $3 a b$ according as $d \equiv \pm 1(\bmod 9)$ or not. Hence, a prime $p \neq 3$ divides $f$ if and only if it divides $d$, if the prime $p=3$ divides $d$ then it divides $f$, and if the prime $p=3$ divides $f$ then it divides $d$ unless $d \not \equiv 0(\bmod 3)$ and $d \not \equiv \pm 1$ (mod 9). Hence, we get the desired result.

Theorem 9. Set $\mathbf{K}_{m}=\mathbb{Q}\left(\sqrt[3]{d_{m}}\right)$ with $d_{m}=m^{3}+1 \geq 2$ cube-free. If $m \geq 3$ then 3 divides the exponent of the ideal class group of $\mathbf{K}_{m}$. Moreover, $\mathbf{K}_{1}, \mathbf{K}_{2}, \mathbf{K}_{3}$ and $\mathbf{K}_{5}$ are the only $\mathbf{K}_{m}$ 's which have ideal class groups of exponents $\leq 3$. Note that $\mathbf{K}_{1}$ and $\mathbf{K}_{2}$ have class number one, that $\mathbf{K}_{3}$ has class number 3 , and that $\mathbf{K}_{5}$ has class number 9 .

Proof. Since $m^{3}+1=(m+1)\left(m^{2}-m+1\right)$ and since any prime divisor $p \neq 3$ of $m^{2}-m+1$ is equal to 1 modulo 3 , then $m \geq 3$ implies that there exists $p \equiv 1(\bmod 3)$ which divides $m^{3}+1$. Let $\mathbf{F}_{p}$ be the cyclic real number field of conductor $p \equiv 1(\bmod 3)$. Then, $\mathbf{F}_{p} \mathbf{K}_{m} / \mathbf{K}_{m}$ is a cyclic cubic unramified (at all the finite and infinite places) extension, which implies that the 3-rank of the ideal class group of $\mathbf{K}$ is $\geq 1$. Indeed, $\mathbf{F}_{p} \mathbf{K}_{m} / \mathbf{K}_{m}$ is clearly unramified outside the prime ideals of $\mathbf{K}_{m}$ lying above $p$, and if it were ramified above some prime ideal $\mathcal{P}$ of $\mathbf{K}_{m}$ lying above $p$, then it would be totally ramified above $\mathcal{P}$ and $\mathbf{F}_{p} \mathbf{K}_{m}(\sqrt{-3}) / \mathbb{Q}(\sqrt{-3})$ would be totally ramified above $p$, which cannot hold. (Let $q \geq 2$ be a prime. If an abelian extension $\mathbf{K} / \mathbf{k}$ with Galois group $\mathbf{Z} / q \mathbf{Z} \times \mathbf{Z} / q \mathbf{Z}$ is totally ramified above some prime ideal $\mathcal{P}$ of $\mathbf{k}$ then $\mathcal{P}$ lies above $q$.) 
Now, according to (2), we have

$$
h_{\mathbf{K}_{m}} \geq \frac{1}{6} \sqrt{d_{\mathbf{K}_{m}} / \log ^{3}\left(3 d_{\mathbf{K}_{m}}\right)}, \quad d_{\mathbf{K}_{m}} \geq 3 \cdot 10^{4} .
$$

For the remainder of this proof we assume that the ideal class group of $\mathbf{K}_{m}$ has exponent 3. Hence, $h_{\mathbf{K}_{m}}=3^{\rho_{3}}$. Let $r$ denotes the number of prime divisors of $d_{m}$ which are not equal to 1 modulo 3 and $s$ denotes the number of prime divisors of $d_{m}$ which are equal to 1 modulo 3 . Then set $\delta_{r, s}=\left(\prod_{i=1}^{r} p_{i}\right)\left(\prod_{j=1}^{s} q_{j}\right)$ where $2=p_{1}<3=p_{2}<5=p_{3}<\cdots$ is the increasing sequence of primes not equal to 1 modulo 3 , and $7=q_{1}<13=q_{2}<\cdots$ is the increasing sequence of primes equal to 1 modulo 3. Then, $\rho_{3} \leq r+2 s$ (see Lemma 8) and $d_{\mathbf{K}_{m}} \geq 3 d_{m}^{2} \geq 3 \delta_{r, s}^{2}$. Set

$$
f(r, s)=\frac{1}{6} 3^{-(r+2 s)} \sqrt{3 \delta_{r, s}^{2} / \log ^{3}\left(9 \delta_{r, s}^{2}\right)}
$$

so that according to (4) we must have $f(r, s) \leq 1$. Since $f(r, 2) \leq f(r, 1) \leq f(r, 0)$ and $f(r, s+1) \geq f(r, s)$ for all $(r, s)$ such that $s \geq 2$, and since $f(r+1, s) \geq f(r, s)$ for all $(r, s)$ such that $r \geq 3$, then $f(r, s) \leq 1$ first implies $r \leq 6$ (since $r \geq 7$ implies $f(r, s) \geq f(7, s) \geq f(7,2)>1$ ), second implies $r+2 s \leq 17$ (since $r=0,1,2$ or 3 implies $s \leq 7$, since $r=4$ implies $s \leq 6, \cdots$, since $r=6$ implies $s \leq 3$ ), third implies $h_{\mathbf{K}_{m}} \leq 3^{r+2 s} \leq 3^{17}$, and according to (4) finally implies $d_{\mathbf{K}_{m}} \leq 10^{23}$ and $m \leq d_{m}^{1 / 3} \leq\left(d_{\mathbf{K}_{m}} / 3\right)^{1 / 3} \leq 4 \cdot 10^{7}$. Moreover, we must have

$$
3^{T_{m}-1} \geq \frac{1}{6} \sqrt{d_{\mathbf{K}_{m}} / \log ^{3}\left(3 d_{\mathbf{K}_{m}}\right)}
$$

where $T_{m}$ is the number of prime ideals of $\mathbf{L}=\mathbb{Q}(\sqrt{-3})$ which are ramified in $\mathbf{K}_{m} \mathbf{L} / \mathbf{L}$. Let $r_{m}$ denote the number of prime divisors of $d_{m}$ which are not equal to 1 modulo 3 and $s_{m}$ denote the number of prime divisors of $d_{m}$ which are equal to 1 modulo 3. According to Lemma 8, we have

$$
T_{m}= \begin{cases}r_{m}+2 s_{m}+1 & \text { if } m \equiv 1(\bmod 3), \\ r_{m}+2 s_{m} & \text { if } m \neq \equiv 1(\bmod 3) .\end{cases}
$$

Only 87 out of the $m \leq 4 \cdot 10^{7}$ such that $d_{m}=m^{3}+1$ is cube-free satisfy this inequality (6), the largest ones being $m=699,803,901$ and 11093 . We note that in these last three cases we have $d_{m} \not \equiv 1(\bmod 9)$ and according to the remarks after Theorem 7 , using $p=5$, we get that these three $\mathbf{K}_{m}$ 's do not have ideal class groups of exponents 3 . Hence, there are only 84 values of $m$ left, the largest one being $m=699$. Finally, only 7 out of these 84 pure cubic fields have class numbers $h_{m}$ which are powers of 3 , namely $m \in\{1,2,3,5,6,11,69\}$. The computation of the 
ideal class groups $H_{m}$ of the corresponding $\mathbf{K}_{m}$ 's yields:

$$
\begin{array}{lll}
m & h_{m} & H_{m} \\
1 & 1 & \\
2 & 1 & \\
3 & 3 & \mathbf{Z} / 3 \mathbf{Z} \\
5 & 3^{2} & (\mathbf{Z} / 3 \mathbf{Z})^{2} \\
6 & 3^{3} & (\mathbf{Z} / 9 \mathbf{Z}) \times(\mathbf{Z} / 3 \mathbf{Z}) \\
11 & 3^{3} & (\mathbf{Z} / 9 \mathbf{Z}) \times(\mathbf{Z} / 3 \mathbf{Z}) \\
69 & 3^{6} & (\mathbf{Z} / 27 \mathbf{Z}) \times(\mathbf{Z} / 3 \mathbf{Z})^{3}
\end{array}
$$

Hence, we get the desired result.

\section{Lower bounds on the exponents of the ideal class groups of the Ishida's cubic number fields}

Set $P_{l}(X)=X^{3}-l X^{2}-1$ where $l \geq 1$ is a positive integer. Then $P_{l}(X)$ is irreducible, has only one real root $\epsilon_{l}$ (and we have $\epsilon_{l}>l$ ) and has negative discriminant $-d_{l}$ with $d_{l}=4 l^{3}+27$. Set $\mathbf{K}_{l}=\mathbb{Q}\left(\epsilon_{l}\right)$. Hence $\mathbf{K}_{l}$ is a non-normal cubic number field. Note that the normal closure $\mathbf{N}_{l}=\mathbf{K}_{l}\left(\sqrt{-d_{l}}\right)$ of $\mathbf{K}_{l}$ is sextic dihedral, and we let $\mathbf{L}_{l}$ be the only quadratic subfield of $\mathbf{N}_{l}$. Hence, $\mathbf{L}_{l}=\mathbb{Q}\left(\sqrt{D_{\mathbf{K}_{l}}}\right)=$ $\mathbb{Q}\left(\sqrt{-d_{l}}\right)$. Since $-d_{l} \equiv 1(\bmod 4)$, then $d_{\mathbf{L}_{l}}$ divides $d_{l}$ and $D_{\mathbf{L}_{l}} \equiv 1(\bmod 4)$.

These fields $\mathbf{K}_{l}$ have been investigated by Ishida. He proved that $\epsilon_{l}$ is the fundamental unit of the cubic order $\mathbf{A}_{l}=\mathbf{Z}\left[\epsilon_{l}\right]$. In [Lou3, Lemma D] we proved that $\mathbf{A}_{l}$ is equal to the ring of algebraic integers of $\mathbf{K}_{l}$ if and only if $3^{2}$ does not divide $l$ and $q^{2}$ does not divide $d_{l}$ for all primes $q \geq 5$. From now on, we will assume that $\mathbf{A}_{l}$ is the ring of algebraic integers of $\mathbf{K}_{l}$. In [Lou3] we used the explicit lower bound

$$
h_{\mathbf{K}_{l}} \geq \frac{\sqrt{d_{l}}}{20 \log ^{2} d_{l}}, \quad\left(d_{l} \geq 2 \cdot 10^{5}\right)
$$

to solve the class number one, two and three problems for these Ishida's real cubic number fields. Here we consider the following ideal class group problem for these number fields : state lower bounds on the exponent of their ideal class groups, and solve the exponent 2 or 3 class group problems.

Lemma 10. Assume that $\mathbf{A}_{l}$ is equal to the ring of algebraic integers of $\mathbf{K}_{l}$. Then,

(i) 3 is the only prime which may be totally ramified in $\mathbf{K}_{l}$, and 3 is totally ramified in $\mathbf{K}_{l} / \mathbb{Q}$ if and only if 3 divides $l$.

(ii) A prime $p$ which is inert in $\mathbf{L}_{l} / \mathbb{Q}$ is neither inert nor totally ramified in $\mathbf{K}_{l} / \mathbb{Q}$, and a prime $p$ which is inert in $\mathbf{K}_{l} / \mathbb{Q}$ splits completely in $\mathbf{L}_{l} / \mathbb{Q}$. 
Proof. Proof of point (i). A prime $p$ is totally ramified in $\mathbf{K}_{l} / \mathbb{Q}$ if and only if $P_{l}(X)$ has a triple root in the finite field with $p$ elements. Proof of point (ii). Since the normal extension $\mathbf{N}_{l} / \mathbb{Q}$ is not cyclic, then $p$ is not inert in $\mathbf{N}_{l} / \mathbb{Q}$, hence not inert in $\mathbf{K}_{l} / \mathbb{Q}$. If $p$ were totally ramified in $\mathbf{K}_{l} / \mathbb{Q}$, we would have $p=3$ and $3 \mid l$. Set $l=3 m$. We would get $d_{l}=3^{3}\left(4 m^{3}+1\right)$. Hence, if 3 does not divide $4 m^{3}+1$ then 3 divides $d_{\mathbf{L}_{l}}$ which contradicts the fact that 3 is inert in $\mathbf{L}_{l} / \mathbb{Q}$. Hence, 3 divides $4 m^{3}+1$, i.e., $m \equiv-1(\bmod 3)$ and setting $m=-1+3 n$ we get $d_{l}=3^{4}(-1+12 t)$ with $t=3 n^{3}-3 n^{2}+n$, which yields $D_{\mathbf{L}_{l}} \equiv 1(\bmod 3)$. Hence, 3 splits completely in $\mathbf{L}_{l} / \mathbb{Q}$, and we get the desired contradiction.

\section{A. Lower bounds on the exponents of the ideal class groups of} $\mathbf{K}_{l}$.

Proposition 11. Assume that $l \geq 9$. If $\alpha \in \mathbf{Z}\left[\epsilon_{l}\right]$ then either there exists a rational integer $n$ and $a$ unit $\eta$ of $\mathbf{Z}\left[\epsilon_{l}\right]$ such that $\alpha=n \eta$, or $\left|N_{\mathbf{K}_{l} / \mathbf{Q}}(\alpha)\right| \geq l$. Note that $\left|N_{\mathbf{K}_{l} / \mathbf{Q}}\left(1-\epsilon_{l}\right)\right|=\left|P_{l}(1)\right|=$ l. In particular, if $\mathbf{A}_{l}=\mathbf{Z}\left[\epsilon_{l}\right]$ is equal to the ring of algebraic integers of $\mathbf{K}_{l}$, then $\operatorname{Min}_{\mathbf{K}_{l}}=l$.

Proof. Since $\mathbf{Z}\left[\epsilon_{l}\right]=\mathbf{Z}+\mathbf{Z} \epsilon_{l}+\mathbf{Z} \epsilon_{l}^{2}=\mathbf{Z}+\mathbf{Z} \epsilon_{l}+\mathbf{Z}\left(\epsilon_{l}^{2}-l \epsilon_{l}\right)=\mathbf{Z}+\mathbf{Z} \epsilon_{l}+\mathbf{Z} \epsilon_{l}^{-1}$, let $\alpha=a+b \epsilon_{l}+c \epsilon_{l}^{-1}$ in $\mathbf{Z}\left[\epsilon_{l}\right]$ be such that $\left|N_{\mathbf{K}_{l} / \mathbf{Q}}(\alpha)\right|=N<l<\epsilon_{l}$. Let $\lambda>0$ be given. Multiplying $\alpha$ by a suitable power of $\epsilon_{l}$ we may assume that we have $\lambda \leq|\alpha|<\lambda \epsilon_{l}$. Let $\alpha, \alpha^{\prime}$ and $\alpha^{\prime \prime}=\bar{\alpha}^{\prime}$ be the three conjugates of $\alpha$ in the cubic number field $\mathbf{K}_{l}$. When $\alpha, \beta$ and $\gamma$ are in $\mathbf{K}_{l}$, we let $d(\alpha, \beta, \gamma)$ denote their discriminant. Solving

$$
\left\{\begin{array}{l}
a+b \epsilon_{l}+c \epsilon_{l}^{-1}=\alpha \\
a+b \epsilon_{l}^{\prime}+c \epsilon_{l}^{\prime-1}=\alpha^{\prime} \\
a+b \epsilon_{l}^{\prime \prime}+c \epsilon_{l}^{\prime \prime}-1=\alpha^{\prime \prime}
\end{array}\right.
$$

and noticing that

$$
\left|\begin{array}{ccc}
1 & \epsilon_{l} & \epsilon_{l}^{-1} \\
1 & \epsilon_{l}^{\prime} & \epsilon_{l}^{\prime-1} \\
1 & \epsilon_{l}^{\prime \prime} & \epsilon_{l}^{\prime \prime-1}
\end{array}\right|^{2}=d\left(1, \epsilon_{l}, \epsilon_{l}^{-1}\right)=d\left(1, \epsilon_{l}, \epsilon_{l}^{2}-l \epsilon_{l}\right)=d\left(1, \epsilon_{l}, \epsilon_{l}^{2}\right)=-d_{l}
$$

we get

$$
b= \pm \frac{i}{\sqrt{d_{l}}}\left|\begin{array}{ccc}
1 & \alpha & \epsilon_{l}^{-1} \\
1 & \alpha^{\prime} & \epsilon_{l}^{\prime-1} \\
1 & \alpha^{\prime \prime} & \epsilon_{l}^{\prime \prime-1}
\end{array}\right| \quad \text { and } \quad c= \pm \frac{i}{\sqrt{d_{l}}}\left|\begin{array}{ccc}
1 & \epsilon_{l} & \alpha \\
1 & \epsilon_{l}^{\prime} & \alpha^{\prime} \\
1 & \epsilon_{l}^{\prime \prime} & \alpha^{\prime \prime}
\end{array}\right|
$$

which yields

$$
|b|=\left|\left(\alpha \epsilon_{l}^{\prime-1}+\alpha^{\prime} \epsilon_{l}^{\prime \prime-1}+\alpha^{\prime \prime} \epsilon_{l}^{-1}\right)-\left(\alpha \epsilon_{l}^{\prime \prime-1}+\alpha^{\prime} \epsilon_{l}^{-1}+\alpha^{\prime \prime} \epsilon_{l}^{\prime-1}\right)\right| / \sqrt{4 l^{3}+27}
$$


and

$$
|c|=\left|\left(\alpha \epsilon_{l}^{\prime \prime}+\alpha^{\prime} \epsilon_{l}+\alpha^{\prime \prime} \epsilon_{l}^{\prime}\right)-\left(\alpha \epsilon_{l}^{\prime}+\alpha^{\prime} \epsilon_{l}^{\prime \prime}+\alpha^{\prime \prime} \epsilon_{l}\right)\right| / \sqrt{4 l^{3}+27} .
$$

Now, we note that we have $1=N_{\mathbf{K}_{l} / \mathbf{Q}}\left(\epsilon_{l}\right)=\epsilon_{l}\left|\epsilon_{l}^{\prime}\right|^{2}=\epsilon_{l}\left|\epsilon_{l}^{\prime \prime}\right|^{2}$, which we write

$$
\left|\epsilon_{l}^{\prime}\right|=\left|\epsilon_{l}^{\prime \prime}\right|=1 / \sqrt{\epsilon_{l}}
$$

and $N=\left|N_{\mathbf{K}_{l} / \mathbf{Q}}(\alpha)\right|=|\alpha|\left|\alpha^{\prime}\right|^{2}=|\alpha|\left|\alpha^{\prime \prime}\right|^{2}$, which we write

$$
\left|\alpha^{\prime}\right|=\left|\alpha^{\prime \prime}\right|=\sqrt{N /|\alpha|} \leq \sqrt{N / \lambda}
$$

Now, $P_{l}\left(\sqrt[3]{l^{3}+(27 / 4)}\right)>0$ implies $\sqrt[3]{l^{3}+(27 / 4)}>\epsilon_{l}$ and $\sqrt{4 l^{3}+27} \geq 2 \epsilon_{l}^{3 / 2}$. Hence, using (9)-(12), we get

$$
|b| \leq \lambda+\frac{1}{\epsilon_{l}} \sqrt{\frac{N}{\lambda}}+\frac{1}{\epsilon_{l}^{5 / 2}} \sqrt{\frac{N}{\lambda}}
$$

and

$$
|c| \leq \sqrt{\frac{N}{\epsilon_{l} \lambda}}+\frac{\lambda}{\epsilon_{l}}+\frac{1}{\epsilon_{l}^{2}} \sqrt{\frac{N}{\lambda}} .
$$

Assume $N \leq l$. Since $9 \leq l<\epsilon_{l}$, the choice $\lambda=1.7$ in (13) and (14) yields $c=0$ and $b= \pm 1$. Now, $\left|N_{\mathbf{K}_{l} / \mathbf{Q}}\left(a-\epsilon_{l}\right)\right|=\left|P_{l}(a)\right|$ and $\left|N_{\mathbf{K}_{l} / \mathbf{Q}}\left(a+\epsilon_{l}\right)\right|=\left|P_{l}(-a)\right|$ are clearly equal to 1 or greater than or equal to $l$, and we get the desired result. Indeed, $a \mapsto P_{l}(a)$ increases on $\left.)-\infty, 0\right]$, decreases on $\left[0, \frac{2 l}{3}\right]$, increases on $\left[\frac{2 l}{3},+\infty(\right.$, and we have $P_{l}(-1)=-l-2<-l, P_{l}(0)=-1, P_{l}(1)=-l, P_{l}(2)=-4 l+7 \leq-l$ (since $l \geq 3$ ), $P_{l}(l-1)=-l^{2}+2 l-2 \leq-l$ (since $l \geq 2$ ), $P_{l}(l)=-1$ and $P_{l}(l+1)=l^{2}+2 l \geq l$.

Theorem 12. Assume that $l \geq 9$ and that $\mathbf{A}_{l}=\mathbf{Z}\left[\epsilon_{l}\right]$ is the ring of algebraic integers of $\mathbf{K}_{l}$. Let $e_{l}$ be the exponent of the ideal class group of $\mathbf{K}_{l}$ and $p$ be a prime such that $p^{e_{l}}<l$. Then, either $p$ is totally ramified in $\mathbf{K}_{l}$, in which case $p=3$ and 3 divides $l$ and $e_{l}$, or $p$ is inert in $\mathbf{K}_{l}$ and splits completely in $\mathbf{L}_{l}$. Hence, under the assumption of the generalized Riemann hypothesis for the imaginary quadratic number fields $\mathbf{L}_{l}$ we have a conditional explicit lower bound $e_{l} \gg \log l / \log \log l$ according which $e_{l} \leq 3$ implies $l \leq 2 \cdot 10^{12}$. Finally, if $l$ is even then we have the unconditional explicit lower bound $e_{l} \geq \log l / \log 2$.

Proof. Let $p$ be a prime which is not inert in $\mathbf{K}_{l}$. Then, there exists a prime ideal $\mathcal{P}$ of $\mathbf{K}_{l}$ of norm $p$. Since $\mathcal{P}^{e_{l}}$ is principal, then either $\mathcal{P}^{e_{l}}$ is trivial or $p^{e_{l}} \geq \operatorname{Min}_{\mathbf{K}_{l}}=l$. Hence, if $p^{e_{l}}<l$ then $\mathcal{P}^{e_{l}}$ is trivial, $p=3$ and 3 is totally ramified in $\mathbf{K}_{l}$ (Lemma 1), and 3 divides $l$ (Lemma 10). Conversely, if 3 divides $l$, 
then $(3)=\mathcal{P}^{3}$ is totally ramified in $\mathbf{K}_{l}$ (Lemma 10 ) and $N(\mathcal{P})=3<l=\operatorname{Min}_{\mathbf{K}_{l}}$. Thus, $\mathcal{P}$ is not principal, the ideal class of $\mathcal{P}$ has order 3 in the ideal class group of $\mathbf{K}_{l}$ and 3 divides $e_{l}$. Since according to Lemma 10 a prime which is inert in $\mathbf{K}_{l}$ splits in $\mathbf{L}_{l}$, we do get the first part of our theorem.

Now, under the assumption of $G R H_{\mathbf{L}_{l}}$ there exists an inert prime $p \leq 3 \log ^{2} d_{\mathbf{L}_{l}}$ in $\mathbf{L}_{l}$ (see [Bac, Th. 3]). Since we have $p^{e_{l}} \geq l$, we get $\left(3 \log ^{2} d_{l}\right)^{e_{l}} \geq\left(3 \log ^{2} d_{\mathbf{L}_{l}}\right)^{e_{l}} \geq$ $p^{e_{l}} \geq l$, which yields

$$
e_{l} \geq \log l / \log \left(3 \log ^{2} d_{l}\right) \gg \log l / \log \log l .
$$

According to this conditional lower bound $e_{l} \geq \log l / \log \left(3 \log ^{2} d_{l}\right)$, then $e_{l}=1$ implies $l \leq 2 \cdot 10^{3}, e_{l}=2$ implies $l \leq 10^{8}$, and $e_{l}=3$ implies $l \leq 2 \cdot 10^{13}$. Moreover, we can improve this last upper bound to $l \leq 2 \cdot 10^{12}$ since [Bac, Table 2] yields that $d_{l} \geq 10^{20}$ implies that there exists a prime $p \leq\left(1.302 \log d_{l}-1.249\right)^{2}$ which is inert in $\mathbb{Q}\left(\sqrt{-d_{l}}\right) / \mathbb{Q}$.

Finally, if $l$ is even, then $P_{l}(X)=(X-1)\left(X^{2}+X+1\right)$ in $Z / 2 Z[X]$ and $(2)=\mathcal{P}_{1} \mathcal{P}_{2}$ in $\mathbf{K}_{l}$ with $\mathcal{P}_{1}=2 \mathbf{A}_{l}+\left(\epsilon_{l}-1\right) \mathbf{A}_{l}$ such that $N_{\mathbf{K}_{l} / \mathbf{Q}}\left(\mathcal{P}_{1}\right)=2$. Hence, Theorem 2 provides us with the desired result.

3B. Conditional solution of the exponent one, two and three class group problems for $\mathbf{K}_{l}$. If $e_{l} \leq 3$ then $2 \leq p<l^{1 / 3}$ and $\left(-d_{l} / p\right) \neq+1$ implies $p=3$ and $3 \mid l$. Let $2=p_{1}<3=p_{2}<\cdots<p_{m}<\cdots$ be the increasing sequence of primes. For each $m \leq 40$, the following table provides the value of $l_{m}$ which is the smallest $l \geq 1$ such that if there exists $i \in\{1, \cdots, m\}$ with $\left(-d_{l} / p_{i}\right)<>+1$, then $i=2$ and $3 \mid l$.

$\begin{array}{llllllll}m & l_{m} & m & l_{m} & m & l_{m} & m & l_{m} \\ 1 & 1 & 11 & 3767 & 21 & 751737 & 31 & 11803374801 \\ 2 & 3 & 12 & 16991 & 22 & 751737 & 32 & 11803374801 \\ 3 & 11 & 13 & 16991 & 23 & 106298361 & 33 & 43239100527 \\ 4 & 11 & 14 & 21281 & 24 & 113532197 & 34 & 149715025821 \\ 5 & 51 & 15 & 21281 & 25 & 277888377 & 35 & 265737999501 \\ 6 & 51 & 16 & 53391 & 26 & 413506937 & 36 & 265737999501 \\ 7 & 147 & 17 & 751737 & 27 & 554996121 & 37 & 265737999501 \\ 8 & 147 & 18 & 751737 & 28 & 554996121 & 38 & 607243185117 \\ 9 & 147 & 19 & 751737 & 29 & 554996121 & 39 & 3444895195911 \\ 10 & 147 & 20 & 751737 & 30 & 6648669141 & 40 & 3444895195911\end{array}$

TheOREM 13. Assume that $\mathbf{A}_{l}$ is the ring of algebraic integers of $\mathbf{K}_{l}$, and let $e_{l}$ be the exponent of the ideal class group of $\mathbf{K}_{l}$. Then, $\mathbf{K}_{l}$ has class number one if and only if $l \in\{1,2,3,5,11\}$. Moreover, $e_{l} \leq 3$ and $l \geq 3 \cdot 10^{5}$ imply $l>3 \cdot 10^{12}$. 
Hence, $e_{l}=2$ and $l \leq 3 \cdot 10^{12}$ if and only if $l \in\{4,7\}$, in which cases $\mathbf{K}_{l}$ has class number 2 , and $e_{l}=3$ and $l \leq 2 \cdot 10^{12}$ if and only if $l \in\{6,15,17\}$, in which cases $\mathbf{K}_{l}$ has class number 3 , or $l=51$ in which case $\mathbf{K}_{l}$ has class number 9 . Finally, under the assumption of the generalized Riemann hypothesis for imaginary quadratic number fields, there are only these 11 number fields $\mathbf{K}_{l}$ with ideal class groups of exponents $e_{l} \leq 3$.

Proof. First, if $\mathbf{K}_{l}$ has class number one problem then according to (8) we have $l \leq 400$, and according to Theorem 12 the cubic polynomial $P_{l}(X)=$ $X^{3}-l X^{2}-1$ has no root in the finite field with $p$ elements for all the prime $p$ which satisfy $2 \leq p<l$. Now, there are only five values of $l$ which satisfy this necessary condition. Namely,

$$
l \in\{1,2,3,5,11\} .
$$

Conversely; for these five values of $l$ the the ring of algebraic integer of $\mathbf{K}_{l}$ is equal to $\mathbf{Z}\left[\epsilon_{l}\right]$ and $\mathbf{K}_{l}$ has class number one (see [Lou3]). Note that the use of Proposition 11 enables us to solve the class number one problem in a much more efficient way than we first solved it in [Lou3], where we had to compute the class number of all the $\mathbf{K}_{l}$ 's with $l \leq 400$. The reader will find in [Lou4] other examples where we use powerful restrictive necessary conditions for class number and class group problems to drastically reduce the amount of required class number computation.

Second, assume $e_{l}=2$ or 3 . Then, according to the previous Table and to Theorem 12 , if $l \geq 3 \cdot 10^{5}$ then $l^{1 / 3}>61=p_{18}$. Hence, we get $l \geq l_{18}=751737$ and $l^{1 / 3}>89=p_{24}$. Hence, we get $l \geq l_{24}=113532197$ and $l^{1 / 3}>479=p_{92}$. Hence, we finally get $l \geq l_{92} \geq l_{39}>3 \cdot 10^{12}$.

(1) Now, there are only 15 values of $l$ with $l \leq 3 \cdot 10^{5}$ such that $P_{l}(X)$ has no root in the finite field with $p$ elements for all primes $p$ such that $2 \leq p<l^{1 / 2}$. Namely,

$$
l \in\{1,2,3,4,5,7,9,11,17,23,41,47,71,107,137\} .
$$

Conversely, for only 14 values out of these 15 values of $l$ we get that $\mathbf{Z}\left[\epsilon_{l}\right]$ is the ring of algebraic integer of $\mathbf{K}_{l}$. Namely,

$$
l \in\{1,2,3,4,5,7,11,17,23,41,47,71,107,137\} .
$$

The computation of the class numbers $h_{l}$ of these 14 number fields $\mathbf{K}_{l}$ (see [Lou3]) yields only 4 values out of them such that the class number of $\mathbf{K}_{l}$ is a positive power of 2. Namely,

$$
l \in\{4,7,23,47\} .
$$

Finally, the computation of the structure of the ideal class groups $H_{l}$ of these 4 
number fields yields the following result:

$$
\begin{array}{lll}
l & h_{l} & H_{l} \\
4 & 2 & \mathbf{Z} / 2 \mathbf{Z} \\
7 & 2 & \mathbf{Z} / 2 \mathbf{Z} \\
23 & 4 & \mathbf{Z} / 4 \mathbf{Z} \\
47 & 8 & (\mathbf{Z} / 2 \mathbf{Z}) \times(\mathbf{Z} / 4 \mathbf{Z})
\end{array}
$$

(2) There are only 187 values of $l$ with $l \leq 3 \cdot 10^{5}$ such that if $P_{l}(X)$ has at least one root in the finite field with $p$ elements for some prime $p$ such that $2 \leq p<l^{1 / 3}$, then $p=3$ and $3 \mid l$. The largest one is $l=70121$. Conversely, for only 153 values out of these 187 values of $l$ we get that $\mathbf{Z}\left[\epsilon_{l}\right]$ is the ring of algebraic integer of $\mathbf{K}_{l}$, the largest one being $l=70121$. The computation of the class numbers of these 153 number fields $\mathbf{K}_{l}$ (see [Lou3]) yields only 5 values out of them such that the class number of $\mathbf{K}_{l}$ is a positive power of 3 . Namely, $l \in\{6,15,17,51,561\}$, in which cases we have

$$
\begin{array}{lll}
l & h_{l} & H_{l} \\
6 & 3 & \mathbf{Z} / 3 \mathbf{Z} \\
15 & 3 & \mathbf{Z} / 3 \mathbf{Z} \\
17 & 3 & \mathbf{Z} / 3 \mathbf{Z} \\
51 & 3^{2} & (\mathbf{Z} / 3 \mathbf{Z})^{2} \\
561 & 3^{5} & (\mathbf{Z} / 81 \mathbf{Z}) \times(\mathbf{Z} / 3 \mathbf{Z})
\end{array}
$$

3C. Suggestions for an unconditional solution of the exponent two class group problem for $\mathbf{K}_{l}$. According to Theorem 12 , if $\mathbf{K}_{l}$ has an ideal class group of exponent 2 , then the primes $p$ less than $d_{\mathbf{L}_{l}}^{1 / 6} \leq d_{l}^{1 / 6} \ll l^{1 / 2}$ are not inert in the imaginary quadratic number field $\mathbf{L}_{l}=\mathbb{Q}\left(\sqrt{-d_{l}}\right)$. However, for any $\epsilon>0$, if $d$ is prime then $n(d)$ the least quadratic non-residue modulo $d$ satisfies $n(d)=O\left(d^{\frac{1}{4 \sqrt{e}}+\epsilon}\right)$ and $\frac{1}{4 \sqrt{e}}<\frac{1}{6}$. Hence, for solving the exponent two class group problem for Ishida's cubic number fields $\mathbf{K}_{l}$ it would be sufficient to have an effective version of Burgess' result which would hold for non necessarily prime conductors.

\section{References}

[ B ] D.A. Burgess, The distribution of quadratic residues and non-residues, Mathematika, 4 (1957), 106-112.

[ Bac ] E. Bach, Explicit bounds for primality testing and related problems, Math. Comp., 55 (1990), 355-380.

[ BK ] D.W. Boyd and H. Kisilevsky, On the exponent of the ideal class groups of complex quadratic fields, Proc. Amer. Math. Soc., 31 (1972), 433-436.

[ Coh ] H. Cohen, A course in computational algebraic number theory, Grad Texts Math., 138, Springer-Verlag, 1993. 
[ Ger ] F. Gerth III, Ranks of 3-class groups of non galois cubic fields, Acta Arith., 30 (1976), 307-322.

[ Hoo ] C. Hooley, On the power free values of polynomials, Mathematika, 27 (1967), 21-26.

[ Ish ] M. Ishida, Fundamental units of certain algebraic number fields, Abh. Math. Sem. Univ. Hamburg, 39 (1973), 245-250.

[ LLS ] D.H. Lehmer, E. Lehmer and D. Shanks, Integers sequences having prescribed quadratic character, Math. Comp., 24 (1970), 433-451.

[Lou1] S. Louboutin, The exponent 2-class group problem for non-Galois over $\mathbf{Q}$ quartic fields that are quadratic extensions of an imaginary quadratic field, J. Number Theory, 49 (1994), 133-141.

[Lou2] S. Louboutin, Une remarque sur l'exposant du groupe des classes d'idéaux des corps cubiques purs, C. R. Acad. Sci. Paris, 320 (1995), 1161-1163.

[Lou3] S. Louboutin, Class-number problems for cubic number fields, Nagoya Math. J., 138 (1995), 199-208.

[Lou4] S. Louboutin, Powerful necessary conditions for class number problems, Math. Nachr., 183 (1997), 173-184.

[ Pap ] F. Pappalardi, On the exponent of the ideal class group of $\mathbf{Q}(\sqrt{-d})$, Proc. Amer. Math. Soc., 123 (1995), 663-671.

[ Ric ] C. Ricci, Ricerche arithmetiche sui polinomi, Rend. Circ. Mat. Palermo, 57 (1933), 433-475.

[Rud] R. Rudman, On the fundamental unit of a purely cubic field, Pacific J. Math., 46 (1973), 253-256.

[ Wei ] P.J. Weinberger, Exponents of the class group, Acta Arith., 22 (1973), 117-124.

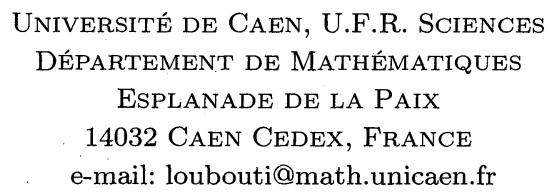

